\title{
¿QUEERIZANDO LA INSTITUCIÓN FAMILIAR? ENTRE LOS DISCURSOS BIO-SOCIALES Y LAS MÚLTIPLES RESISTENCIAS
}

\author{
Gracia TRUjiLlo \\ Universidad de Castilla-La Mancha \\ ELENA BURGALETA \\ Universidad Complutense de Madrid
}

Recibido: 24/03/2014

Aceptado: 12/05/2014

\section{Resumen}

Este artículo analiza la(s) maternidad(es) desde una perspectiva feminista y queer, tratando de responder a la pregunta de hasta qué punto las comaternidades lesbianas reproducen el modelo heteronormativo de familia o lo ponen en cuestión, transformándolo. Comenzamos con un recorrido sobre la construcción socio-histórica de la maternidad en Occidente, incluyendo las aportaciones que, desde los diversos feminismos, se han realizado sobre cuestiones como la sexualidad, la reproducción y el parentesco. El análisis empírico está centrado en las parejas de lesbianas que utilizan las técnicas de reproducción asistida en el contexto español actual; para conocer sus opiniones, inquietudes, dilemas, etc., y recoger sus voces realizamos veinte entrevistas en profundidad con ellas. El trabajo finaliza con una serie de propuestas que sugerimos para continuar los análisis, reflexiones y diálogos tan necesarios sobre este tema.

Palabras clave: Teorías y prácticas feministas, maternidades, sexualidades, reproducción y parentesco, matriz heteronormativa, comaternidades lésbicas, técnicas de reproducción asistida, renaturalización.

\section{Abstract}

This article analyses motherhood(s) from a feminist and queer perspective, trying to answer the question as to what extent lesbian co-mothers reproduce or rather question the heteronormative family model. We begin with a socio- historical revision 
about the construction of motherhood in Western cultures, including the most relevant contributions made by different feminist perspectives about issues such a as sexuality, reproduction and kinship. The empirical analysis focuses on lesbian couples which have used assisted reproduction techniques in Spain; in order to know their opinions, concerns and dilemmas and gather their voices, we carried out twenty in depth interviews. This study concludes with some proposals the authors' suggest should be looked into, in order to further the analysis, reflections and much needed dialogues about this issue

Keywords: Feminist theories and practices, maternities, sexualities, reproduction and kinship, heteronormative matrix, lesbian co-mothers, assisted reproduction techniques, renaturalization. 


\section{Introducción: La construcción socio-histórica de la maternidad en occidente}

Tradicionalmente, la maternidad ha sido estudiada por las Ciencias Sociales como un elemento más del orden social y cultural; fue en la década de los setenta cuando los movimientos feministas comenzaron a denunciar el carácter androcéntrico de los estudios históricos, antropológicos, sociológicos y psicológicos, cuestionando la homogeneidad de la categoría madre y proponiendo nuevas y diversas perspectivas teóricas, entre ellas otro análisis histórico. El mito del matriarcado originario ha contado, entre las feministas, tanto con defensoras como con detractoras. Beauvoir, por ejemplo, consideraba que la defensa de la existencia de pasado matriarcal y la consecuente pérdida del derecho materno ancestral venía a respaldar los argumentos acerca de la supuesta incapacidad «natural» de las mujeres para ejercer poder ${ }^{1}$. Por otro lado, autoras como Adrienne Rich o Luce Irigaray interpretan el estudio del poder femenino en la Antigüedad como instrumento de revalorización de la relación maternal usurpada por el sistema patriarcal ${ }^{2}$. No hay, por tanto, un consenso entre las feministas sobre la cuestión de la existencia o no de un matriarcado primitivo; sin embargo, sí parece haber acuerdo en el análisis de la relación entre la aparición de la ley escrita y el establecimiento de las primeras expresiones patriarcales. Así, por ejemplo, el primer código legal conocido, el Código de Hammurabi (1760 A.C.), ya recogía castigos de pena de muerte para las mujeres adúlteras, y en las denominadas culturas pre-clásicas (sumerias, hebreas y egipcias principalmente) se defendían las prácticas de sometimiento hacia las mujeres.

La forma principal en que las hebreas podían ser valoradas era teniendo hijos, por lo que su peor desgracia era la esterilidad, que recaía sobre la instancia divina y quien tenía el poder de otorgarla o retirarla. Se trata, por lo

1. Beauvoir de, Simone. El Segundo sexo. Volumen I: Los hechos y los mitos. Madrid, Cátedra, 1949/1986.

2. Rich, Adrienne. Nacida de mujer. La crisis de la maternidad como institución y como experiencia. Barcelona, Noguer, 1978, e IRIGARAY, Luce. El cuerpo a cuerpo con la madre. Barcelona, La Sal, 1981. 
tanto, de lo que Anna Goldamn-Amirav define como la «operación de apropiación de la capacidad reproductora por parte del Dios patriarcal», donde el papel de las mujeres se centra única y exclusivamente en la producción de descendientes varones ${ }^{3}$. En Grecia las mujeres no sólo fueron marginadas del espacio político, sino que además fueron denigradas, definidas como la parte pasiva de la reproducción, argumento que servirá para justificar su inferioridad social. La cultura romana se apropió de esta concepción de la superioridad masculina: el pater familias será la figura principal de la familia romana, el dueño de las personas y de los bienes que la componen, el único que no está subordinado a ninguna otra autoridad ${ }^{4}$. El último período del Imperio Romano supondrá el inicio de un proceso de idealización de la maternidad que culminará en la Edad Media con el ensalzamiento de la figura de la Virgen. La tradición católica creó un modelo de mujer-madre que, en su pasividad, no peca porque no tiene deseos sexuales.

A lo largo de los siglos XVIII y XIX, el liberalismo político, el capitalismo económico y el racionalismo científico dieron lugar a un nuevo orden social que supondrá, entre otros cambios, una revalorización del individuo-varón. La mayoría de los ideólogos del contrato social, Hobbes, Locke o Rousseau, entre otros, coinciden en definir la reproducción humana como una «cuestión de las mujeres». Siguiendo esta lógica será precisamente la «naturaleza» de las mujeres la causa de su estado de sumisión y el marco en el que se conceptualizó la existencia del amor maternal natural. No hay que olvidar que el surgimiento de esta ideología del amor maternal coincide con el interés del Estado moderno por aumentar los índices de natalidad tras las guerras del siglo XVIII en Europa. En este marco nació la familia burguesa, que tomará forma a lo largo del siglo XIX, organizada en torno a la mujer-madre procreadora, que no ciudadana, y la maternidad pasó a ser objeto de discurso público e institución legal. Con la «legalización» de la maternidad en el siglo XIX se reconcilian las dos esferas que conformaban a la madre: la mujer de la «naturaleza» y la mujer de la «sociedad», dando lugar a la madre-educadora. La madre burguesa llevará a cabo un importante «servicio a la patria», como gestadora y educando a los futuros ciudadanos (y a las futuras madres).

En el s. XIX, la ciencia médica no sólo utilizará sus recursos científicos, sino que hará uso asimismo de elementos del pensamiento mítico, lo cual le permitirá posicionarse como grupo portador de un sub-universo de

3. Goldamn-AmiraV, Anna. «Mira, Yahveh me ha hecho estéril», en Silvia Tubert (ed.): Figuras de madre, Madrid, Cátedra, 1996, p. 45.

4. Mulliez, Jacques. «La Volonté d'un homme», en Jean Delumeau y Daniel Roche (eds.): Histoire des pères et de la paternité, Paris, Larousse, 1990, pp.83-103. 
significados con un alto poder de control social. Michel Foucault denominará a la posición de superioridad incuestionable de la ciencia en la sociedad moderna el nuevo poder/saber ${ }^{5}$. Durante el Antiguo Régimen el poder/saber sólo muestra interés por la muerte, es el poder del soberano quien ejerce el derecho sobre la vida en cuanto que es capaz de decidir acerca de la muerte de sus súbditos. A partir del siglo XVIII, en cambio, el poder se va a ejercer sobre la vida convirtiéndose en lo que el teórico francés definió como biopoder, que se desarrolla y expande a través de una serie de tecnologías que comienzan a formarse en la modernidad, cuando la teoría política incorpora el problema de «protección de la vida» como fundamento de la sociedad ${ }^{6}$. La jerarquía entre las personas que investigan frente a las que les asisten quedaron establecidas ya a principios del siglo XX; así, entre 1900 y 1930, las comadronas cayeron bajo la hegemonía de la profesión médica, perdiendo su función autónoma de sanadoras.

\section{La maternidad desde los feminismos. Igualdad y diferencia en España}

La cuestión de la maternidad ha sido, para la mayor parte de los feminismos, un debate cerrado, o, cuando menos, una cuestión de segundo orden. Harriet Taylor fue la primera en analizar la maternidad como el principal argumento para desacreditar a las mujeres como ciudadanas: «Es una descalificación injusta hacer imperativo a las mujeres que han de ser madres o ser nada, o que, si una vez que han sido madres, no serán nada más durante el resto de sus vidas» ${ }^{7}$. Fue en la década de los 60 y 70 cuando la cuestión de la maternidad toma finalmente relevancia en los debates feministas, aunque en la obra de Simone de Beauvoir ya encontramos algunas claves para el análisis:

Existe una mala fe extravagante que pretende conciliar el desprecio que se siente por las mujeres y el respeto con el que se rodea a las madres. Es una paradoja criminal negar a la mujer toda actividad pública, cerrarle las carreras masculinas, proclamar su incapacidad a los cuatro vientos y confiarle la empresa más delicada, la más grave que pueda existir: la formación de un ser humano ${ }^{8}$.

5. FouCAult, Michel. Historia de la sexualidad. Vol.1. La voluntad de saber. Madrid, Siglo XXI, 1987.

6. FouCAUlt, Michel. Arqueología del saber. Madrid, Siglo XXI, 1969/2006.

7. MiLL, John Stuart y Harriet Taylor. Ensayos sobre la igualdad sexual. Madrid, Cátedra, 1869/2001, p. 129.

8. BEAuvoir de, Simone. El Segundo sexo. Volumen II: La experiencia vivida. Madrid, Cátedra, 1949/1986, p. 321.

Feminismo/s 23, junio 2014, pp. 159-179 
No obstante, otras autoras feministas como Adrienne Rich o Luce Irigaray encontrarán en la relación madre-hija una fuente de placer, conocimiento y poder específicamente femeninos. Rich definió la imposición del modelo heterosexual como la máxima expresión del patriarcado moderno. La negación de la existencia lesbiana es, según la autora, el fundamento esencial en la construcción de una sociedad heteronormativa que se impone desde la infancia a través del ideal romántico. Crítica con el antibiologicismo de Beauvoir, Rich reclama implícitamente un retorno a la biología en su necesidad de una revalorización de la maternidad, entendida como opción y no como institución, distinguiendo así entre maternal thinking (maternidad como experiencia), vivida como fundamento del poder creativo en la relación entre mujeres, y maternal practice, es decir, la maternidad como institución patriarcal sustentadora del orden de dominación masculina ${ }^{9}$. Siguiendo las propuestas de Rich, Luce Irigaray considera que el significado del cuerpo de la madre viene dado a través del contacto físico, el cual permite a la niña establecer su primer sentimiento de amor y deseo. Es, por lo tanto, primordial recuperar esa relación primaria y arcaica que permitirá descubrir la singularidad del autoerotismo entre mujeres ${ }^{10}$. Las propuestas de Irigaray tendrán en la década de los 80 y 90 un gran impacto entre las feministas italianas centradas en el estudio del orden simbólico de la madre como Luisa Muraro, entre otras.

Estos dos posicionamientos teóricos tuvieron su correlato en el feminismo español en dos líneas de pensamiento diferenciadas y habitualmente enfrentadas: el feminismo «de la igualdad»y «de la diferencia». Influenciado por el feminismo francés e italiano, el feminismo de la diferencia en España se inspira, por una parte, en la crítica de Irigaray a un pensamiento patriarcal que margina y oprime esa diferencia, y por otra, en las italianas y su apuesta por el affidamento y la figura de la madre simbólica como pilares de la conformación de una identidad esencialmente femenina. Los principales postulados de la diferencia en España se pueden analizar a través de dos de sus principales teóricas: Victoria Sendón de León y Milagros Rivera Garretas. Sendón de León, partiendo de las propuestas de Julia Kristeva, propone recuperar el simbolismo del renacimiento y de la regeneración que nos remite a la madre. Esta recuperación del «orden sagrado de la madre» conllevará, según la autora, la liberación de hombres y mujeres de la socialización diferenciada ${ }^{11}$. Por otro lado, Rivera Garretas defiende que la sociedad actual está inmersa en un desorden

9. RiCH, Adrianne. Op. cit.

10. IRIGARAY, Luce. Op. cit.

11. SENDÓN DE LEÓN, Victoria. Marcar las diferencias: discursos feministas ante un nuevo siglo. Barcelona, Icaria, 2002. 
simbólico generado por la anulación patriarcal de la figura materna, a lo que se añade la propia animadversión del feminismo de la igualdad hacia la figura de la madre ${ }^{12}$. Victoria Sau, cercana a los planteamientos de la diferencia, parte de la idea del matricidio originario de Irigaray ${ }^{13}$, denunciando el vacío de la maternidad, resultado de una ideología paternal que ha secuestrado y sustituido la maternidad originaria por una simple labor de maternaje (mothering). Sau distingue así entre una madre distante y fría, la madre patriarcal, de una madre originaria, prepatriarcal o real a la que denomina madre entrañable. A pesar de mostrarse crítica con la «naturalización» del amor maternal, Sau predice un futuro en el que la desacralización del patriarca permitirá la revalorización de la madre y, por tanto, de las mujeres en su conjunto ${ }^{14}$.

Crítica con estos postulados, Celia Amorós asegura que estas propuestas refuerzan una visión dicotómica de la realidad, al potenciar las relaciones entre mujeres y la transmutación simbólica de la valoración de las identidades y los espacios. De esta manera la exaltación de la madre que caracteriza al feminismo (o, mejor, los feminismos) de la diferencia legitima el pacto patriarcal a través del nuevo pacto que representa la leche materna ${ }^{15}$. En esta misma línea, la filósofa Alicia Puleo propone revisar cómo la construcción de la madre en el pensamiento occidental ha conjugado simultáneamente un discurso «del elogio» $\mathrm{y}$ «del desprecio». Puleo habla también de una nueva «moral del vientre» para referirse a la revalorización de la maternidad que actualmente proponen algunos feminismos postmodernos y que, en su opinión, supone un regreso a modelos idealizados en torno a la triada mujer-madre-naturaleza ${ }^{16}$.

\section{Feminismos Queer}

Podemos afirmar, evocando la célebre frase de Beauvoir, que «las madres no nacen, se hacen»; y a esto hay que sumar que no todas las madres se autodefinen como mujeres: pueden ser lesbianas butch (o femme), intersexuales,

12. Rivera Garretas, María Milagros. Nombrar el mundo en femenino. Barcelona, Icaria, 1994.

13. IRIGARAY, Luce. Op. cit.

14. SAU, Victoria. El vacío de la maternidad. Madre no hay más que ninguna. Barcelona, Icaria, 1995.

15. Amorós, Celia. «Discurso de la diferencia, discurso de la igualdad». El viejo topo, 10, 1980, pp. 30-33.

16. Puleo, Alicia H. «Perfiles filosóficos de la maternidad», en Ángeles de la Concha Muñoz y Raquel Osborne (eds.): Las mujeres y los niños primero, Barcelona, Icaria, 2004 , pp. 32-45. 
o trans masculinos, entre otras ${ }^{17}$. Autoras como Gayle Rubin, Kath Weston y Judith Butler han realizado una serie de aportaciones críticas, desde una mirada no heteronormativa, que han sido clave en el análisis de las sexualidades, la reproducción, el parentesco y la familia. Para estas tres teóricas, como apunta Libson, «desmontar la heterosexualidad implica poner en cuestión los presupuestos clásicos de la antropología, el psicoanálisis y la sociología, y pensar lo familiar desde un lugar radicalmente diferente» ${ }^{18}$.

La antropóloga Gayle Rubin llamó la atención, a comienzos de los años ochenta, sobre cómo las sexualidades no heterosexuales son construidas socialmente como extrañas y desviadas, y situadas en una jerarquía sexual con diferentes niveles de visibilidad y respetabilidad. Esta jerarquía sexual divide el sexo en «bueno» (natural, normal, saludable) y «malo» (antinatural, anormal, patológico) y establece entre ambos extremos una serie de fronteras sexuales que marcan la virtud y el vicio, el orden sexual y el caos. En el borde de la respetabilidad, como apunta Rubin, están las parejas estables de gays y lesbianas, «seguidos en el descenso hacia el sexo "malo" por los gays y lesbianas promiscuos, hasta llegar a los niveles más bajos de la jerarquía sexual, los más estigmatizados: prostitutas, travestis, transexuales, sadomasoquistas, fetichistas, etc» ${ }^{19}$. El lesbianismo, en concreto, ha sido, a lo largo de la Historia (y continúa en numerosas partes del planeta a día de hoy), invisibilizado, perseguido y sometido a diferentes tipos de violencias por formar parte de las denominadas sexualidades periféricas, fuera de la norma, del régimen de la heterosexualidad obligatoria.

Los sujetos con otras opciones sexuales diferentes a la norma heterosexual se enfrentan, por tanto, a mayores violencias, a menor visibilidad, legitimidad, respetabilidad y reconocimiento legal, a la presunción de enfermedad mental, y a la pérdida de apoyo institucional. Las penalizaciones sociales a las y los disidentes de la norma heterosexual son todavía algo a no perder de vista incluso en los contextos en los que hemos ido ganando algunos derechos y libertades, gracias, sobre todo, a la movilización colectiva en la calle durante

17. Uno de los casos más mediáticos estos últimos años ha sido el del embarazo del trans masculino Thomas Beatie en 2008. Véase su artículo «Labour of love. Is society ready for this pregnant husband?», en http://www.advocate.com/news/2008/03/14/laborlove? page=full, consultado el 7-04-2014.

18. LiBSON, Micaela Cynthia. La diversidad en las familias: un estudio social sobre parentalidad gay y lesbiana. Buenos Aires, 2009. http://inadi.gob.ar/wp-content/uploads/2010/04/ Libson_La-diversidad-en-las-familias.pdf, consultado el 7/2/14.

19. RUBIN, Gayle. «Reflexionando sobre el sexo: notas para una teoría radical de la sexualidad», en Carole Vance (comp.): Placer y peligro. Explorando la sexualidad femenina, Madrid, Talasa, 1984, p. 308. 
varias décadas. Una cuestión central aquí es que no entrar en las categorías de la heteronormatividad constituye un riesgo, una probabilidad mayor de ser discriminado/a, pero también un cambio epistemológico fundamental, como señala Teresa De Lauretis:

Rechazar el contrato heterosexual, no sólo en la práctica de vida de cada una sino en la práctica de conocimiento,-lo que Wittig denominó una «práctica cognitiva, subjetiva»-, constituye un giro epistemológico ya que cambia las condiciones de posibilidad del saber y del conocimiento, y esto constituye un giro en la conciencia histórica ${ }^{20}$.

Judith Butler se referirá a la matriz heterosexual como el «entramado de inteligibilidad cultural mediante la que los cuerpos, géneros y deseos son naturalizados ${ }^{21}$, concepto basado, como señala ella misma, en el contrato heterosexual de Monique Wittig, y, en menor medida, en el concepto de heterosexualidad obligatoria de Adrianne Rich. Un ejemplo de la presunción de heterosexualidad son los términos monoparentalidad o el más reciente monomarentalidad, definidos en relación a la «ausencia» del otro progenitor (y aquí se entiende comúnmente que es el padre en el caso de las familias monomarentales encabezadas por una mujer, que son la mayoría, o la madre en el supuesto de las mantenidas por un varón), hecho que invisibiliza a las madres y padres homosexuales. Esta presunción de heterosexualidad, sin embargo, se puede (y así se hace) utilizar estratégicamente, en los casos de adopción y/o fertilización asistida, por parte de gays y lesbianas para acceder a la materni$\mathrm{dad} /$ paternidad en contextos de legislaciones que no reconocen a las familias que se escapan del marco heteronormativo.

Ante las dificultades y hostilidades mencionadas anteriormente (muchas veces provenientes de las propias familias de origen), lesbianas, gays, bisexuales y transexuales (LGTB, de aquí en adelante) van construyendo otras formas de familias, que Kath Weston ha denominado familias elegidas, es decir, aquellas que no se vinculan biológicamente, sino que se construyen a través de relaciones de amistad e identificación entre los grupos discriminados ${ }^{22}$. Esta propuesta considera que la familia no es una institución estática, sino una categoría cultural, flexible que debe representar para la comunidad LGTB y

20. LAURETIS, de Teresa. «When lesbians were not women», en revista Labrys, études feminists. Número especial, Septiembre, 2003, p. 14.

21. BUTLER, Judith. El género en disputa. El feminismo y la subversión de la identidad. México, Paidós, 1990/2001, p. 5.

22. Weston, Kath. Families We Choose: Lesbians, Gays, Kinship. Nueva York, Columbia University Press, 1991. 
queer más un desafío que un instrumento de asimilación. Como ha señalado asimismo Judith Butler en relación al parentesco en Deshacer el género:

El parentesco pierde su especificidad como objeto una vez que se lo caracteriza aproximadamente como los modos de relaciones duraderas. Obviamente, no todas las relaciones de parentesco duran, pero cualquiera que sean las relaciones que se califican como parentesco entran dentro de una norma o una convención que tiene alguna duración y esa norma perdura al ser reiterada una y otra vez. Así pues, una norma no tiene que ser estática para perdurar; de hecho no puede ser estática si tiene que durar ${ }^{23}$.

Weston (1991), en su estudio sobre relaciones familiares de gays y lesbianas, analiza cómo habitualmente la familia biológica de origen tiene un papel menos importante que el de las familias elegidas, es decir, las constituidas a partir de redes de amistad y que asumen muchas de las funciones que tradicionalmente se espera de los familiares. Para esta autora, la relación con la familia de origen juega un papel fundamental en el desarrollo de las identidades, pero, si hay un rechazo por la condición sexual, la «comunidad» suple el rol de apoyo afectivo y emocional. Por esa razón, y como mostró en su estudio realizado en San Francisco en los 80, era habitual que gays y lesbianas se refirieran a su grupo de amigos/as como su familia ${ }^{24}$. Nuestras entrevistas, realizadas en el contexto español ${ }^{25}$, más de dos décadas después, van en la línea del trabajo de Weston. Algunas muestras son las siguientes:

$\mathrm{Si}$, sobre todo, amigos, porque con mi madre ya te digo que bien, pero no como para que se venga a mi casa, no. Me siento mucho más cómoda con amigos (Noemí, 36).

Es cierto que nuestras familias están y no están (...) les visitamos cuando queremos, es decir, que no tenemos a nuestras familias en nuestras vidas (...) mis amigas, que es otra forma, otra familia... (Elsa, 40).

23. Butler, Judith. Deshacer el género. Barcelona, Paidós, 2006, p. 54.

24. Weston, Kath, Op. cit.

25. Los datos aquí presentados forman parte de una investigación sobre la maternidad y los nuevos modelos de familias. Los datos fueron recogidos durante el trabajo de campo realizado en en áreas urbanas y rurales de las Comunidades Autónomas de Andalucía, Cantabria, Cataluña, Extremadura, Madrid, Murcia, Navarra, País Vasco y Valencia entre 2011 y 2012. Durante el mismo, se realizó un total de 20 entrevistas en profundidad. Todas las informantes eran mujeres que se definían como lesbianas y que en el momento de la investigación formaban parte de familias co-marentales, las cuales estaban criando niñas y niños que habían nacido a través de técnicas de reproducción asistida. Todas las entrevistas fueron grabadas y transcritas, manteniendo el anonimato y la confidencialidad de las participantes, razón por la cual aquí hemos modificado los nombres originales. 
Preguntas como las que se formula Butler, « $i$ decimos que las familias que no se aproximan a la norma, pero reflejan la norma de alguna manera aparentemente derivativa, son copias baratas, o aceptamos que la identidad de la norma es desarticulada precisamente a través de la complejidad de su puesta en escena? ${ }^{26}$ son, como adelantamos en la introducción, las que guían nuestro análisis en este trabajo: los cambios en los ámbitos de la sexualidad, la reproducción y el parentesco, ¿qué transformaciones sociales están generando?; ¿de qué tipo, estructurales o más bien superficiales?; ¿se reproduce el modelo heteronormativo de pareja y familia o estamos modificándolo?; ¿cuáles son las diversas resistencias que pueden o desean articular otras formas de familias no heterosexuales?.

\section{3. ¿«Nuevas» familias? Comaternidades lesbianas}

En los años setenta y ochenta, los grupos de gays y lesbianas analizaron y debatieron las consecuencias de defender un modelo familiar no heterosexual. Mientras algunas voces, las más críticas o radicales, subrayaban el peligro de que la identificación entre familia y heterosexualidad diera lugar a una asimilación al modelo heteronormativo, otras, más moderadas, defendían que el parentesco gay y lésbico ofrecía una alternativa legítima e independiente. Iris Young se encuentra entre las autoras que defendió deconstruir el matrimonio y llevar a cabo una revolución cultural dirigida hacia una política más justa de pluralismo familiar para construir la familia plural. Frente al concepto de matrimonio, su propuesta es la de un modelo de «compañerismo doméstico» (domestic partnership) que pueda ser universalizado, que implique derechos y obligaciones y en el que el sexo no sea la condición necesaria ni suficiente para su conformación ${ }^{27}$. Por otra parte, Judith Stacey prefiere hablar de familia postmoderna, un nuevo modelo que refleje los cambios de la sociedad actual: las madres solteras, las familias reconstituidas, las parejas de hecho o las parejas de gays y lesbianas ${ }^{28}$. Esta socióloga ha subrayado el papel de los feminismos en la conformación de estos «nuevos» ${ }^{29}$ tipos de familia al defender el

26. ButLer, Judith. El grito de Antígona. Barcelona, El Roure, 2001, p. 106.

27. Young, Iris Marion. «Mothers, Citizenship, and Independence: A Critique of Pure Family Values». Ethics, 105 (1995), pp. 535-556.

28. STACEY, Judith (1990) Brave New Families: Stories of Domestic Upheaval in Late Twentieth-Century America, Nueva York: Basic Books.

29. Entrecomillamos «nuevos» para señalar que no se trata de formas de organización familiar que no existían previamente (pensemos en las hijas e hijos que se criaban con los abuelos/as al emigrar los progenitores, las madres solteras, las parejas que cohabitan sin casarse, las familias reagrupadas...), sino que en las últimas décadas empiezan a ser más visibles y a demandar y conseguir el reconocimiento legal y social. 
divorcio y ser una fuente de empoderamiento y de apoyo para las mujeres que se tienen que enfrentar a la crianza sin pareja, entre otras cuestiones.

El trabajo de Robert William Connell hace hincapié en la idea de que la familia es esencialmente una «fábrica de heterosexualidad», que sirve para satisfacer «la necesidad del capital de trabajo y la necesidad del Estado para la subordinación ${ }^{30}$ Matrimonio y familia son, según el autor, productos y agentes de un régimen heterosexual cuyo objetivo principal es la represión del deseo homosexual. Siguiendo la propuesta de Connell, Dennis Altman insiste en que las personas no heterosexuales representan en sí mismas un claro rechazo a la institución familiar y que ésta es precisamente la causa de su persecución: la necesidad de mantener la hegemonía heteropatriarcal ${ }^{31}$. En este punto coincidirá la socióloga feminista Elizabeth M. Ettorre, que defiende que las identidades gay, lesbianas y trans niegan en sí mismas la primacía de la familia ${ }^{32}$. Así, el parentesco es un símbolo de asimilación y marca el límite entre la identidad heterosexual y el resto.

En este punto conviene recordar que, en nuestro país y hasta hace relativamente poco tiempo, lesbianas, transexuales, gays, travestis... han sido considerados «peligrosos sociales» y susceptibles de incurrir en delito de «escándalo público». En 1970 (un año después de la Revuelta de Stonewall, que marcó la reemergencia de los movimientos de liberación sexual en los países occidentales), la Ley de Peligrosidad y Rehabilitación Social (LPRS) convirtió en «peligrosos sociales» al conjunto de los y las no heterosexuales. La LPRS vino a sustituir a la Ley de Vagos y Maleantes, de 1954, que, a su vez, era heredera de la instaurada durante la Segunda República, en 1933; esta última, no obstante, no incluía a «los homosexuales». Anteriormente, la Dictadura de Primo de Rivera sí había sancionado legalmente la homosexualidad en el Código Penal de 1928. La LPRS no fue derogada hasta 1979, mientras que el delito de escándalo público se mantuvo hasta $1988^{33}$.

En el contexto español, los temas relativos a las sexualidades, la reproducción y el parentesco han estado controlados y vigilados de cerca por la Iglesia católica y los discursos médicos y legales. Como mostramos al inicio del artículo, desde la modernidad se ha vinculado a las mujeres, desde diferentes

30. CONNELL, Robert William. Gender and Power. Stanford: University Press, 1987.

31. Altman, Dennis Patkin. Coming Out in the Seventies. Syndey, Wild \& Woolley, 1979.

32. ETtOrRE, Elizabeth M. Lesbians, Women and Society. Londres, Routledge \& Kegan Paul, 1980.

33. Véase De FluviÁ, Armand. «El movimiento homosexual en el Estado español», en Enríquez, J.R (comp.) El homosexual ante la sociedad enferma. Barcelona, Tusquests, 1978, y TrujILlo, Gracia. Deseo y resistencia. Treinta años de movilización lesbiana en el Estado español. Madrid, Egales, 2009. 
instancias, con la naturaleza y con la reproducción. El cuerpo de las mujeres, como ha señalado Preciado ha sido disciplinado para ser maternal ${ }^{34}$. Una de las demandas del movimiento feminista ha sido precisamente la separación de los ámbitos de la sexualidad y la reproducción -algo que ya habían defendido las feministas socialistas, en términos de explotación-, reivindicando el placer y el control por parte de las mujeres de sus propios cuerpos y vidas (pensemos en el acceso a métodos anticonceptivos, el divorcio, el aborto libre y gratuito, la legitimidad de otras opciones sexuales distintas a la norma heterosexual, la lucha contra las agresiones sexistas...). Los derechos sexuales y reproductivos han sido históricamente, y son aún, un campo de batalla, y, como sabemos, este no es un tema cerrado, sino que se trata más bien una lucha sin fin: en la actualidad, volvemos a salir a la calle para frenar los ataques a los derechos de las mujeres (como el proyecto restrictivo de Ley del aborto presentado por el Partido Popular y actualmente en trámite parlamentario $)^{35}$.

\section{Comaternidades, naturaleza y tecnología}

El trabajo de Ellen Lewin sobre madres lesbianas es uno de los que sostiene que, frecuentemente, la comaternidad cumple una función «naturalizadora» ${ }^{36}$. De esta manera, la maternidad «natural» es un mecanismo que se enfrenta al hecho de que las sexualidades no normativas hayan sido tradicionalmente definidas como desviadas, «antinaturales» o «contra natura». En otras palabras, debido a estos discursos con una larga andadura histórica, existe un conflicto entre la identidad de lesbiana y madre. Así, aquellas mujeres que habían sido expulsadas de la «naturaleza» por su sexualidad anormal, se encuentran con la posibilidad de reconciliarse con su «ser mujeres» a través de esta «renaturalización». Son lesbianas pero son madres: cumplen, por lo tanto, con el mandato bio-social. Recordando a Rubin y su jerarquía sexual, las lesbianas monógamas y en pareja tienen un estatus un poco más alto que las «lesbianas en el bar $»^{37}$. Las lesbianas madres no tienen y no alcanzarán probablemente nunca el mismo estatus que las heterosexuales (probablemente muchas ni

34. Entrevista realizada por Úrsula del Águila en 2009: «Judith Butler et Beatriz Preciado en entretien», http://tetu.yagg.com/2009/08/14/archives-tetu-judith-butler-et-beatrizpreciado-en-entretien-3/, consultado el 30/3/2014.

35. A este respecto: «El Gobierno aprueba la ley del aborto más restrictiva de la democracia»,http://sociedad.elpais.com/sociedad/2013/12/20/actualidad/1387544028 _883233.html, consultado el 27/3/2014.

36. LEWIN, Ellen. Lesbian Mothers. Accounts of gender in American culture. Londres, Cornell University Press, 1993.

37. RuBin, Gayle. Op. cit.

Feminismo/s 23, junio 2014, pp. 159-179 
siquiera persigan esa homologación), pero, en líneas generales, se puede decir que ganan algo en respetabilidad social. Los dilemas y conflictos identitarios y vitales entre ser lesbiana (no mujer, o mujer no «natural»y, por tanto, no madre) y querer ser madre (y conseguirlo) se traducen, en muchas ocasiones, en el eclipsamiento de la identidad lesbiana, o su paso a un segundo plano:

Te identificas ahora más con esas madres, con lo que pasas, con lo que sufres (...) Yo antes de..., sí, era partidaria de un poco el 'ghetto', cuando era 'bollo' individual. Una vez que ya he sido madre, no considero que tenga que andar identificándome como tal (Beatriz, 36).

El total de las veinte lesbianas entrevistadas comparten la idea del deseo maternal en términos de instinto, es decir, de acto «natural», no racional; recordemos que los ideólogos del contrato social mencionados utilizaron como argumento esta supuesta naturaleza femenina indomable para dejar a las mujeres fuera de la ciudadanía.

El hecho de que la mayoría se refiera al «instinto maternal»-una construcción socio-cultural, e instrumento de dominación patriarcal tal y como demostró Elizabeth Badinter-, evidencia la dificultad de escapar a las expectativas de género ${ }^{38}$. Recogemos aquí dos ejemplos, a modo de muestra:

Yo quiero ser madre desde que me vino el instinto maternal; me vino super joven, a los 19 o así (Sara, 38).

Es un deseo tan fuerte... Es una maternidad doble, por así decirlo, un instinto aflorado por las dos partes (Noemí, 36).

Este discurso renaturalizador había hecho su aparición ya en la década de los setenta cuando algunas lesbianas empezaron a utilizar las técnicas de reproducción asistida (TRA, de aquí en adelante) ${ }^{39}$. Como apunta Weston, algunos movimientos propusieron sustituir el término inseminación artificial por inseminación alternativa, con el fin de evitar la identificación entre artificial

38. BADINTER, Elizabeth. ¿Existe el instinto maternal? Historia del amor maternal, siglos XVII al XX. Paidós, Barcelona, 1981. En este trabajo, Badinter muestra cómo el «instinto maternal» es una invención creada en el siglo XVIII y desarrollada a lo largo del XIX, para inducir a las mujeres a consagrarse al cuidado de sus hijos. Asimismo, evidencia cómo el concepto «instinto» no se aplica en ningún caso sobre la especie humana, salvo el «instinto maternal» que, aún siendo sustituido por el de «amor maternal», mantiene su significado: un deseo de la mujer «naturalmente» incontrolable.

39. Las RTA son los procedimientos a través de los cuales se introducen los espermatozoides humanos, por un método no coital, en el sistema reproductivo de una (bio) mujer, es decir, un sujeto asignado mujer al nacer, lo que incluiría a transexuales masculinos; entre las TRA se incluyen la fecundación in vitro (FIV), la recepción de óvulos de la pareja (ROPA) y la inseminación, entre otras. 
y no-natural ${ }^{40}$. Judith Butler, en la entrevista mencionada anteriormente, realizada junto a Beatriz Preciado, señala que en la reproducción siempre hay una técnica; esta última, en la misma línea, advierte que ningún embarazo es natural: uso de la píldora, de tratamientos médicos, las cesáreas...y un largo etcétera ${ }^{41}$.

En varias de nuestras entrevistas encontramos la identificación de ser madre con la aceptación de la responsabilidad social y con una especie de demostración de haberse "asentado», frente a la idea de rebeldía y desobediencia asociada a las sexualidades no heteronormativas. Las reconciliaciones familiares, especialmente con la madre, aparecen en las entrevistas como una prueba de madurez. La maternidad se presenta así como el cambio de una vida adolescente, provocadora y caótica, por una adulta y responsable:

Bueno ahora, por ejemplo la relación con mi madre es más equilibrada, en eso ha mejorado. Me trata, vamos, nos tratamos de otra manera, es curioso, es como otro respeto (Elsa, 40).

De esta manera, la reproducción supone una especie de recuperación del amor maternal perdido al haberse saltado (y cuestionado, se quiera o no) la norma heterosexual, que se consigue, precisamente, al reconocer el valor del estatus maternal. De nuevo la re-naturalización -ahora como «re-familiarización»o «re-maternalización»-, se traduce, en líneas generales, en una posibilidad de reconciliación (si así sucede y/o se quisiera) con todo aquello con lo que se había transgredido. En este sentido, una forma de resistencia al modelo heteronormativo que hemos encontrado es la ausencia de intención de homologarse, y/o la reivindicación legitima de no tener que cumplir con ningún mandato de ser buenas madres, en un contexto de ausencia de referentes y modelos (lo que, a su vez, es fuente de numerosos conflictos en las parejas). El denominado «criterio de la excelencia», exigido históricamente a las mujeres que querían acceder a cualquier espacio «masculino» (el ámbito político, entre muchos otros), opera también demasiado a menudo con las familias no heterosexuales, como una especie de mecanismo de compensación de sus «deficiencias» respecto al ser unas madres perfectas (no ser suficientemente mujeres, ser vistas como raras, o, cuando menos, otras mujeres). Si bien no compartimos la perspectiva anti-queer de Ellen Lewin, nos parece acertado el apunte que esta antropóloga hizo al ser entrevistada a este respecto:

Los «fundamentalistas queer» dicen: «iTenemos que ser diferentes, tenemos que resistir!». Eso es muy bonito, pero la gente real no se siente así.

40. WeSTON, Kath. Op. cit.

41. Del ÁGuila, Úrsula. «Judith Butler et Beatriz Preciado en entretien», Op. cit.

Feminismo/s 23, junio 2014, pp. 159-179 
Muchos de ellos quieren tener sus familias, quieren pasar las Navidades con sus padres... Eso es lo que quieren y tenemos que reconocer que esta gente existe y son de verdad. Y que quizás sus deseos y expectativas no son revolucionarias, pero son reales ${ }^{42}$.

Retomando la cuestión de la renaturalización, otro ejemplo de este proceso es el protagonismo que están recuperando estos últimos años los discursos biologicistas; siguiendo la lógica del mercado, las clínicas de fertilización han comenzado a ofertar el tratamiento ROPA (recepción de óvulos de la pareja), consistente en que una de las lesbianas sea inseminada utilizando el óvulo de la otra. De esta manera, una es la «madre gestante» y la otra la «madre biológica ${ }^{43}$. Entre las mujeres entrevistadas, maternidad y pareja suelen aparecer asociadas:

Y al conocer a Eva, pues ella tenía tantas ganas de ser madre como yo (...) Yo quería, pues eso, tener una pareja, formar una familia (Charo, 29).

O sea, mi deseo de ser madre nació cuando la conocí a ella. Sí, sí. (...) Igual también fue conocerla a ella y dejar esa parte como más mental e ir a una parte más corporal. Yo creo que por ahí también se me pudo despertar la maternidad (Judith, 26).

El modelo de pareja sigue teniendo mayor peso (y reconocimiento) en la sociedad en la que vivimos, un modelo que excluye a aquellas personas que optan por una maternidad/paternidad en solitario (monomarentalidad / monoparentalidad). Las parejas de lesbianas no están, como ninguna pareja,

42. «Entrevista a Ellen Lewin», AIBR (Revista de Antropología Iberoamericana), Volumen 2 Número 1. Enero-Abril 2007, p.13. http://www.aibr.org/antropologia/02v01/entrevistas/020101.pdf, consultado el 20/3/14.

43. A pesar de que este procedimiento no incorpora ninguna novedad, es decir, no incorpora ninguna innovación médico-técnica en el campo de las tecnologías reproductivas, supone un coste mínimo de 8.000 euros; este dato lo hemos obtenido a través de personas cercanas que han pasado recientemente, o se encuentran en ello (2014), por algún centro de reproducción asistida. Las páginas de estos centros, como el IVI, no dan esta información. www.ivi.es, consultado el 20/3/2014. Otra cuestión relevante es que España es el tercer país donde más tratamientos de reproducción asistida se realizan cada año, con más de 54.000 ciclos anuales. «España es el tercer país europeo con más tratamientos de fertilidad», en El País: http://sociedad.elpais.com/sociedad/2009/02/17/ actualidad/1234825203_850215.html, consultado el 1/4/14. Sin embargo, no puede conocerse el número de lesbianas ya que no están desagregados, a lo que hay que añadir que se trata de datos estimativos ya que la mayoría de las inseminaciones asistidas o fecundaciones in vitro se realizan en clínicas privadas. De los 203 centros que hay actualmente en España 165, es decir, el 81\%, son privados, según datos de la Asociación pro Derechos Civiles, Económicos y Sociales (ADECES), recogidos en el artículo: «Espera hasta de cuatro años para concebir» www.elpais.com/articulo/sociedad/Espera/anos/ concebir/elpepisoc/20080701elpepisoc_2/Tes?print=1, consultado el 7/4/14. 
exentas de relaciones de poder entre sus integrantes, aunque hay que subrayar que muchos de los problemas y conflictos a los que se enfrentan estas parejas son generados por un sistema heteropatriarcal y capitalista que cuestiona de manera constante a todas las formas de familias que no se adecúen al modelo hegemónico de familia nuclear heterosexual; las familias de lesbianas, transexuales y gays tienen que hacer frente a una serie de violencias, estereotipos y prejuicios como la relativa al posible «contagio» de la homosexualidad a su descendencia, o la falta de un "padre» en el caso de las parejas de lesbianas, entre muchas otras. Estas hostilidades explicarían, en gran parte, que la mayoría de ellas decidan inseminarse en pareja. Desde el punto de vista legal, recientemente hemos visto que las parejas de lesbianas y las mujeres sin pareja han quedado vetadas en la sanidad pública para acceder a los servicios de las TRA, derecho que estaba recogido en la Ley de Reproducción Asistida de $2007^{44}$. La Ministra de Sanidad Ana Mato declaró, para justificar este recorte de derechos, que «la falta de varón no es un problema médico» ${ }^{45}$.

\section{Conclusiones reflexiones y propuestas}

Como hemos tratado de mostrar a lo largo de estas líneas, desde el proyecto moderno la maternidad fue construida de acuerdo a la lógica de la «naturaleza», definida como un espacio irracional e incontrolable en el que quedan atrapadas las mujeres. De ahí que la idea del instinto maternal siga teniendo una fuerte presencia en los discursos dominantes, incluso desde un pensamiento científico que niega la existencia de los instintos humanos, pero que, curiosamente, continúa hablando del «reloj biológico» de las mujeres. Las comaternidades lesbianas se enfrentan también a esta misma lógica natural, por lo que no es extraño observar cierta tendencia a la reproducción, en las voces aquí recogidas, de la idea del instinto maternal, que entra en contradicción con los discursos acerca de las sexualidades no heteronormativas, y, por tanto, desviadas, no naturales. Observamos, así, cómo en las parejas de lesbianas entrevistadas, el ser madres y los procesos de renaturalización que le acompañan se traducen en una tensión entre las identidades de madre y lesbiana, donde frecuentemente el peso de la maternidad (y lo que supone en

44. «Sanidad confirma que las mujeres sólo podrán acceder a la inseminación por razones médicas», en http://www.eldiario.es/politica/sanidad-anamato-fertilidad-reproduccionasistida_0_156784818.html, y «Asturias niega la inseminación a una lesbiana» http:// www.publico.es/espana/372977/asturias-niega-la-inseminacion-a-una-lesbiana, accedidos el 23/3/14.

45. Véase http://www.publico.es/actualidad/459346/mato-no-creo-que-la-falta-de-varonsea-un-problema-medico, accedido el 20/3/14.

Feminismo/s 23, junio 2014, pp. 159-179 
términos de cumplimiento de las expectativas de género y de homologación social) se impone frente a la identidad lesbiana, estigmatizada y más difícil de visibilizar. Por otra parte, hemos observado que las comaternidades tienden a reproducir, en general, el ideal maternal en un marco de familia compuesta por dos personas.

Llegadas a este punto, volvemos sobre las preguntas iniciales relativas a si es posible elaborar estrategias y alternativas al modelo maternal heteronormativo; si pueden ser las comaternidades una oportunidad para no reproducir las instituciones del matrimonio y la familia heteropatriarcales o están condenadas a reproducir el mandato (que no parece, por otra parte, haber perdido en la actualidad tanta fuerza como cabría esperar, o en ocasiones escuchamos). Para responder a estas preguntas, sería interesante recordar las reflexiones iniciadas en las décadas de los 60 y 70 en torno al poder de las instituciones de la familia, la pareja (monógama) y el matrimonio, que fueron, desde los años 90, retomadas por los grupos queer y feministas. El Grupo de Trabajo Queer (GTQ), organizado en Madrid en 2002, es uno de los que reflexionó sobre estas cuestiones, y organizó acciones en torno a estos debates, en el contexto de la aprobación de la modificación del Código civil (2005), que permitió a lesbianas y gays casarse en el estado español ${ }^{46}$. Los grupos queer, entre ellos GTQ, y el Grupo de Lesbianas Feministas (GLF) de Barcelona fueron las únicas voces críticas con aquel proceso, que dejaba de lado la reclamación histórica del movimiento LGTB de una ley de parejas de hecho estatal, y posponía las reivindicaciones de los colectivos trans de una Ley de Identidad de Género, que se aprobaría, finalmente, en $2007^{47}$.

Para continuar reflexionando sobre los dilemas entre el asimilacionismo y las resistencias desde «dentro» (y los espacios intermedios), los análisis de Michel Foucault sobre el poder y la resistencia nos parecen una caja de herramientas especialmente útil. Foucault sostenía que «donde hay poder hay resistencia», lo cual no significa que la última sea la imagen invertida del primero, pero sí que, como el poder, es «tan inventiva, tan móvil, tan productiva como él. Es preciso, por lo tanto, que, como el poder, se organice, se coagule y se cimiente. Que vaya de abajo arriba, como él, y se distribuya

46. «La familia nuclear es radioactiva» fue el eslogan con el que marchamos, lxs integrantes de GTQ y amigxs, en la manifestación del Orgullo de 2005. Véase el libro que editamos, El eje del mal es heterosexual. Figuraciones, movimientos y prácticas feministas queer (Traficantes de sueños, 2005), un libro copy left disponible en el siguiente enlace: http://webs.uvigo.es/pmayobre/pdf/el_eje_del_mal.pdf, consultado el 7/3/2014.

47. TrujILlo, Gracia. Op. cit. 
estratégicamente» ${ }^{48}$. En otras palabras, allí donde se da una relación de poder existe la posibilidad de subversión, de resistencia, y de conceptualizar éstas como procesos de creación y de transformación. $\mathrm{La}(\mathrm{s})$ resistencia(s) se construye sobre la base de la experiencia vivida por aquellxs que hacen de ella una auténtica práctica de libertad. La propuesta foucaultiana de la resistencia puede seguir inspirándonos en la creación y puesta en marcha de otras formas alternativas de organización de la crianza, de los cuidados, los afectos, para seguir trabajando en la construcción de relaciones igualitarias, y escapando del mandato heteronormativo.

En este punto, tampoco deberíamos olvidar que cada experiencia es resultado de un contexto, en el que entran en juego numerosas variables como la clase social, el nivel educativo, los recursos disponibles, el lugar de residencia, la edad o la diversidad funcional, entre otros, que determinan la manera en que esas resistencias se articulan o pueden articularse. Por esta razón, consideramos que, al pensar sobre las maternidades, es necesario tener siempre en cuenta los diferentes contextos y situaciones en las que se viven. En el momento actual de crisis del sistema financiero a nivel global y de recortes sociales sin precedentes en nuestro país, el Gobierno del Partido Popular está inmerso además en un giro ideológico que, entre otras muchas cuestiones y como hemos comentado anteriormente, estamos viendo reflejado en la expulsión de lesbianas y madres solteras de la reproducción asistida en la Sanidad pública o en la restricción del derecho al aborto. Así, se obliga a mujeres que no quieren ser madres a serlo, y a otras que sí querrían se les priva de esa posibilidad, ahondando además las desigualdades sociales: en la actualidad, sólo pueden acceder a las TRA las que tengan recursos para acudir a las clínicas privadas.

En definitiva, desde nuestro posicionamiento queer y feminista nos parece fundamental cuestionar y suprimir, de una vez por todas, la figura de La Madre y todos los discursos que siguen vigentes (incluso, en ocasiones, desde los feminismos) acerca de lo que debe hacer una madre y lo que no. Las maternidades tienen contextos, situaciones, dificultades específicas, que no podemos obviar. En este sentido, las comaternidades lesbianas son muy diversas, y dependen de diferentes factores, como comentábamos anteriormente. Creemos que es más que necesario seguir pensando y conversando sobre las maternidades en general, y las lesbianas en particular, e ideando maneras de resistir al modelo de la buena madre socialmente impuesto, y de la familia

48. FouCAUlt, Michel. «No al sexo rey. Entrevista por Bernard Henry-Levy», en Un diálogo sobre el poder, Barcelona, Altaya, 1994, p. 162. 
heteropatriarcal. En este punto, nos quedan muchas cuestiones por debatir y más dudas que certezas o conclusiones cerradas tras nuestro estudio, pero lo que sí nos parece urgente es que conversemos acerca de las maternidades (y paternidades), de otras formas de organización de los afectos y los cuidados (nuestras «familias elegidas»), y que sigamos defendiendo que todas merecen el mismo respeto y reconocimiento, y que son igualmente legítimas.

\section{Referencias bibliográficas}

Amorós, Celia. «Discurso de la diferencia, discurso de la igualdad». El viejo topo, 10 1980, pp. 30-33

Altman, Dennis Patkin. Coming Out in the Seventies. Syndey, Wild \& Woolley, 1979.

BADINTER, Elizabeth. ¿Existe el instinto maternal? Historia del amor maternal, siglos XVII al XX. Paidós, Barcelona, 1981.

Beauvoir de, Simone. El Segundo sexo. Volumen I: Los hechos y los mitos. Madrid, Cátedra, 1949/1986.

El Segundo sexo. Volumen II: La experiencia vivida. Madrid, Cátedra, 1949/1986.

BUTLER, Judith. El género en disputa. El feminismo y la subversión de la identidad. México, Paidós, 1990/2001.

- El grito de Antígona. Barcelona, El Roure, 2001.

- Cuerpos que importan. Buenos Aires, Paidós, 2002.

- Deshacer el género. Barcelona, Paidós, 2006.

Connell, Robert William. Gender and Power. Stanford: University Press, 1987.

DE FluviÁ, Armand. 1978. «El movimiento homosexual en el Estado español», en Enríquez, J.R (comp.) El homosexual ante la sociedad enferma. Barcelona: Tusquests.

ETTORRE, Elizabeth M. Lesbians, Women and Society. Londres, Routledge \& Kegan Paul, 1980.

FouCAULt, Michel. Arqueología del saber. Madrid, Siglo XXI, 1969/2006.

- Historia de la sexualidad. Vol.1. La voluntad de saber. Madrid, Siglo XXI, 1987.

- «No al sexo rey. Entrevista por Bernard Henry-Levy», en Un diálogo sobre el poder, Barcelona, Altaya, 1994, pp.161-162.

Goldamn-Amirav, Anna. «Mira, Yahveh me ha hecho estéril», en Silvia Tubert (ed.): Figuras de madre, Madrid, Cátedra, 1996, pp.48-56.

IRIGARAY, Luce. El cuerpo a cuerpo con la madre. Barcelona, La Sal, 1981.

LAURETIS de, Teresa. "When lesbians were not women», en revista Labrys, études feminists. Número especial, Septiembre, 2003.

LEWIN, Ellen. Lesbian Mothers. Accounts of gender in American culture. Londres: Cornell University Press, 1993. 
LiBSOn, Micaela Cynthia. La diversidad en las familias: un estudio social sobre parentalidad gay y lesbiana. Buenos Aires, 2009.

MiLl, John Stuart y Harriet TAYLOR. Ensayos sobre la igualdad sexual. Madrid, Cátedra, 1869/2001.

MullieZ, Jacques. «La Volonté d'un homme», en Jean Delumeau y Daniel Roche (eds.): Histoire des pères et de la paternité, Paris, Larousse, 1990, pp.83-103.

Puleo, Alicia H. «Perfiles filosóficos de la maternidad», en Ángeles de la Concha Muñoz y Raquel Osborne (eds.): Las mujeres y los niños primero, Barcelona, Icaria, 2004, pp. 32-45.

Rich, Adrienne. Nacida de mujer: La crisis de la maternidad como institución y como experiencia. Barcelona, Noguer, 1978.

Rivera GARRETAS, María Milagros. Nombrar el mundo en femenino. Barcelona, Icaria, 1994.

RUBIN, Gayle. «Reflexionando sobre el sexo: notas para una teoría radical de la sexualidad», en Carole Vance (comp.): Placer y peligro. Explorando la sexualidad femenina, Madrid, Talasa, 1984.

SAU, Victoria. El vacío de la maternidad. Madre no hay más que ninguna. Barcelona, Icaria, 1995.

SENDÓN DE LEÓN, Victoria. Marcar las diferencias: discursos feministas ante un nuevo siglo. Barcelona, Icaria, 2002.

StaCEY, Judith. Brave New Families: Stories of Domestic Upheaval in Late Twentieth-Century America, Nueva York: Basic Books, 1990.

TrujiLlo, Gracia. Deseo y resistencia. Treinta años de movilización lesbiana en el Estado español. Madrid: Egales, 2009.

Weston, Kath. Families We Choose: Lesbians, Gays, Kinship. Nueva York, Columbia University Press, 1991.

YounG, Iris Marion. «Mothers, Citizenship, and Independence: A Critique of Pure Family Values». Ethics, 105 (1995), pp. 535-556. 